\title{
Early intervention with mesenchymal stem cells prevents nephropathy in diabetic rats by ameliorating the inflammatory microenvironment
}

\author{
YUANMIN LI ${ }^{1}$, JINGPING LIU ${ }^{1}$, GUANGNENG LIAO ${ }^{1}$, JIE ZHANG $^{1}$, YOUNAN CHEN $^{1}$, LAN LI $^{1}$, \\ LI LI $^{1}$, FANG LIU ${ }^{2}$, BO CHEN ${ }^{1}$, GANG GUO ${ }^{1}$, CHENGSHI WANG ${ }^{1}$, LICHUAN YANG ${ }^{2}$, \\ JINGQIU CHENG ${ }^{1}$ and YANGRONG LU ${ }^{1}$
}

\begin{abstract}
${ }^{1}$ Key Laboratory of Transplant Engineering and Immunology, National Health and Family Planning Commission (NHFPC) and ${ }^{2}$ Department of Nephrology, West China Hospital, Sichuan University, Chengdu, Sichuan 610041, P.R. China
\end{abstract}

Received August 20, 2016; Accepted September 1, 2017

DOI: $10.3892 / \mathrm{ijmm} .2018 .3501$

\begin{abstract}
Diabetic nephropathy (DN) is a major complication of diabetes and represents the leading cause of end-stage renal disease. Mesenchymal stem cell (MSC) treatment has been demonstrated to be effective in DN models by reducing albuminuria and attenuating glomerular injury; however, limited in-depth understanding of the underlying mechanism and a lack of clinical trials hinders its clinical use. Additionally, most of these experimental studies were conducted on the advanced stage of nephropathy, which is difficult to reverse and consequently showed limited therapeutic efficacy. We sought to evaluate whether early intervention by MSCs has the potential to prevent $\mathrm{DN}$ onset and progression as well as protect kidney function when intravenously administered to rats with diabetes. Diabetes was induced in adult male SD rats by streptozotocin (STZ) injection ( $55 \mathrm{mg} / \mathrm{kg}$, i.p.). The diabetic rats were injected with or without bone marrow-derived MSCs $\left(5 \times 10^{6}\right.$ per rat), via tail vein at 2, 4, 5 and 7 weeks after diabetes onset. Fasting blood glucose (FBG), blood urea nitrogen (BUN) and serum creatinine (Scr) levels in serum samples and glycosuria (GLU), microalbumin (MAU), and albumin to creatinine ratio (ACR) in urine samples were determined. Renal pathology and immunohistochemistry (IHC) for CD68, MCP-1, fibronectin (FN), transforming growth factor- $\beta$ (TGF- $\beta)$ and pro-inflammatory cytokines were also performed. Expression levels of the above factors as well as interleukin-10 (IL-10), and epidermal growth factor (EGF) were assessed by qPCR and multiplex bead-based suspen-
\end{abstract}

Correspondence to: Dr Yanrong Lu, Key Laboratory of Transplant Engineering and Immunology, National Health and Family Planning Commission (NHFPC), West China Hospital, Sichuan University, Chengdu, Sichuan 610041, P.R. China

E-mail: luyanrong@scu.edu.cn

Key words: bone marrow-derived mesenchymal stem cells, diabetic nephropathy, immunoregulation, macrophages, inflammatory microenvironment sion array system, respectively. Additionally, MSC tracing in vivo was performed. Ex vivo, peritoneal macrophages were co-cultured with MSCs, and expression of inflammatory cytokines was detected as well. MSC treatment profoundly suppressed renal macrophage infiltration and inflammatory cytokine secretion in diabetic rats, resulting in prominently improved kidney histology, systemic homeostasis, and animal survival, although no significant effect on hyperglycemia was observed. Engrafted MSCs were primarily localized in deteriorated areas of the kidney and immune organs $48 \mathrm{~h}$ after infusion. MSC treatment upregulated serum anti-inflammatory cytokines IL-10 and EGF. Ex vivo, MSCs inhibited lipopolysaccharide (LPS)-stimulated rat peritoneal macrophage activation via the downregulation of inflammatory-related cytokines such as IL-6, MCP-1, tumor necrosis factor- $\alpha$ (TNF- $\alpha$ ) and IL-1 $\beta$. Our results demonstrated that early intervention with MSCs prevented renal injury via immune regulation in diabetic rats, which restored the homeostasis of the immune microenvironment, contributing to the prevention of kidney dysfunction and glomerulosclerosis.

\section{Introduction}

Approximately $20-40 \%$ of diabetic patients develop diabetic nephropathy (DN), a clinical syndrome that comprises renal failure and increased risk of cardiovascular disease $(1,2)$. DN is characterized by persistent albuminuria, development of hyperfiltration and histopathological lesions including extracellular matrix deposition, glomerular basement membrane thickening, glomerular and mesangial expansion (3). As DN progresses to end-stage chronic kidney disease, patients would require hemodialysis and even kidney transplant in the end (4).

Multiple factors may contribute to the development and outcomes of DN. For instance, interactions between genetic and environmental factors are likely to determine the susceptibility of DN. Hyperglycemia is the major driving force, and the progressive renal hemodynamic changes may be the leading cause for DN development. Essentially, inflammatory pathways are critical to its progression $(5,6)$. During the last decade, the findings that macrophages infiltrate the kidney and produce a 
pro-inflammatory microenvironment have drawn more attentions in DN research. It is common knowledge that macrophages are central mediators of inflammatory responses $(7,8)$. In most types of human kidney diseases, macrophage accumulation in both glomeruli and interstitial tissues correlates closely with the degree of renal structural injury and renal dysfunction, such as DN (9-11). Additionally, migrated macrophages release multiple cytokines under pathological stimuli, such as TNF- $\alpha$, interleukin-1 $\beta$ (IL-1 $\beta$ ), and IL-6, provoking inflammatory responses through autocrine and paracrine manners, resulting in inflammatory cascades and accelerated renal injury $(12,13)$.

Current treatments, involving glycemic and blood pressure control, can delay the development of DN, but do not stop the progression to end-stage renal failure (14). Novel and effective therapeutic approaches are in serious demand to prevent against nephropathy in patients with early-stage diabetes mellitus (DM).

Cell therapy with mesenchymal stem cells (MSCs) has become an attractive therapeutic strategy with which to regulate the immune response invoked in pathologies, such as tissue injury, transplantation, and autoimmunity (15). The primary characteristics of MSCs are their immunomodulatory ability, capacity for self-renewal, and ability to differentiate into mesodermal tissues (16). Additionally, autologous MSCs are easily harvested and expanded in culture, and are free of immunorejection, implying the promising application in clinical treatment (17). Several studies have shown that MSCs lead to amelioration of acute or chronic renal injury, caused by ischemia reperfusion injury, 5/6 nephrectomy, unilateral ureteral ligation or even DN (18-20). In these studies, the immune modulation and anti-apoptotic effects of MSCs through paracrine mechanisms have been associated with the therapeutic effect (21-23). More importantly, it has been demonstrated that functional interactions occur between MSCs and macrophages (8). The immunomodulatory capacity to inhibit macrophage infiltration is likely to be a critical mechanism in MSC-mediated amelioration of inflammation-related diseases (24).

Based on the previous findings, we transplanted bone marrow-derived MSCs in rats with streptozotocin (STZ)induced diabetes to explore the therapeutic potential of MSCs on renal dysfunction in the early stage of diabetes and disclose its immunoregulatory role on macrophage activity and the inflammatory environment.

\section{Materials and methods}

Rat model of STZ-induced diabetes. Male SD rats ( $\mathrm{n}=30$; age, 10 weeks old; weight, 280-300 g) were purchased from Chengdu Dashuo Laboratory Animal Technology Co. (Chengdu, China) and housed in an animal facility under controlled temperature $\left(23 \pm 1^{\circ} \mathrm{C}\right)$, humidity $(45-65 \%)$, and a 12-h light/dark cycle with free access to water and chow. Diabetes was induced by a single intraperitoneal injection of $55 \mathrm{mg} / \mathrm{kg} \mathrm{STZ} \mathrm{(Sigma}$ Chemical, St. Louis, MO, USA) dissolved in sodium citrate buffer ( $\mathrm{pH} 4.5$ ) after overnight fasting. Three days later, the rats with a fasting glucose level $>16.7 \mathrm{mM}$ for 3 consecutive days were identified as diabetic rats (14). None of the rats received insulin treatment during the entire course of the experiment. Afterwards, the development of renal injury was evaluated via biochemical and histopathological assessment. All experimental protocols and studies were approved by the
Animal Ethics Committee of the Sichuan University, which are consistent with the National Institutes of Health Guide for the Care and Use of Laboratory Animals.

Isolation, culture and characterization of MSCs and macrophages. Bone marrow-derived MSCs were isolated from femurs and tibias of 25 3-week-old male SD rats (100-120 g; purchased from Chengdu Dashuo Laboratory Animal Technology Co.); the rats were sacrificed prior to the isolation of the MSCs. Cells were then cultured in low-glucose Dulbecco's Modified Eagle's Medium (DMEM) containing 10\% fetal bovine serum (FBS) (Gibco), $100 \mathrm{U} / \mathrm{ml}$ penicillin, and $100 \mathrm{mg} / \mathrm{ml}$ streptomycin $(25,26)$. The surface markers of MSCs were identified by flow cytometric analysis using fluorophore conjugated antibodies: anti-rat CD29-FITC, CD44-FITC, CD34-PE and CD45-PE (BD Biosciences, Franklin Lakes, NJ, USA). For multiple differentiation, MSCs were induced to differentiate into adipocytes and osteocytes for 14 and 21 days in adipogenic and osteogenic media (Cyagen Biosciences Inc., Santa Clara, CA, USA), respectively. Oil Red stain for lipid droplets and Alizarin Red for calcium deposition were then used. Furthermore, 5-ethynyl-2'-deoxy-uridine (EdU) staining was performed to confirm the proliferation capacity of MSCs before transplantation. The method of collecting rat peritoneal macrophages via intraperitoneal injection of phosphate-buffered saline (PBS) was carried out according to a previous study (27). Briefly, 10 healthy 12-week-old rats (400-450 g; purchased from Chengdu Dashuo Laboratory Animal Technology Co.) were administered a lethal dose of sodium pentobarbital (Nembutal, $100 \mathrm{mg} / \mathrm{kg}$ ) to induce deep anesthesia; subsequently, $20 \mathrm{ml}$ of the cold harvest medium was injected into the peritoneal wall via syringe needle (21-G). Under sterile condition, a small incision was made on the abdomen and abdominal lavage fluid was collected. The peritoneal exudate cells (PECs) were then centrifuged at $400 \mathrm{x} \mathrm{g}$ for $10 \mathrm{~min}$ and washed twice with sterile PBS. To determine the purification of isolated macrophages, cells were stained with PE-conjugated anti-mouse CD68 antibody, F4/80 and flow cytometry was performed via a Coulter ${ }^{\circledR}$ Epics $^{\circledR}$ $\mathrm{XL}^{\mathrm{TM}}$ cytometer (FC500; Beckman Coulter, Brea, CA, USA).

\section{In vivo experiments}

Experimental design and MSC transplantation. A total of 30 rats were randomly assigned into 3 groups as follows: i) normal control group $(\mathrm{NC}, \mathrm{n}=5)$, healthy rats without treatment; ii) DN group ( $n=14)$, diabetic rats receiving injection of $0.5 \mathrm{ml} 0.9 \%$ saline instead of MSCs; iii) DN-MSC group $(\mathrm{n}=11)$, diabetic rats with MSC transplantation. Subsequently, 2, 4, 5 and 7 weeks after successful establishment of the diabetes model, MSCs were transplanted via the tail vein at a concentration of $5 \times 10^{6}$ cells in $0.5 \mathrm{ml} 0.9 \%$ saline.

Biodistribution of transplanted MSCs. A total of 810 -weekold male SD rats (280-300 g) were purchased from Chengdu Dashuo Laboratory Animal Technology Co., and T1D models were obtained by a single intraperitoneal administration of $55 \mathrm{mg} / \mathrm{kg}$ of STZ. To track the MSCs after transplantation, MSCs were labeled with CM-DiI (Invitrogen Life Technologies, Carlsbad, CA, USA) according to the manufacturer's instructions. Briefly, the third passage of MSCs were incubated with CM-DiI in a working solution $(1 \mu \mathrm{g} / \mathrm{ml})$ for $5 \mathrm{~min}$ at $37^{\circ} \mathrm{C}$, followed by a further $15 \mathrm{~min}$ incubation at $4^{\circ} \mathrm{C}$. After washing twice with 


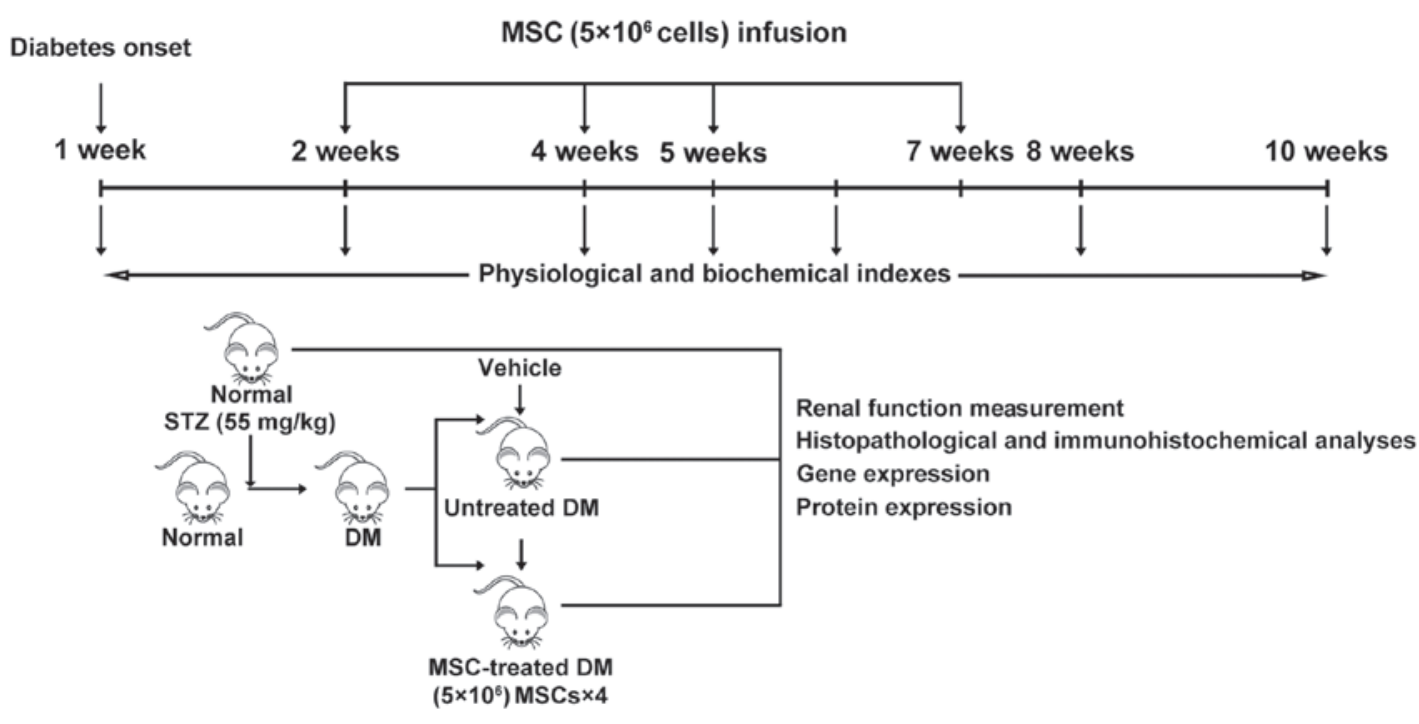

Figure 1. Experimental design.

Table I. Primer sequences used for quantitative PCR.

\begin{tabular}{ll}
\hline Target & \multicolumn{1}{c}{ Primer sequences $\left(5^{\prime} \rightarrow 3^{\prime}\right)$} \\
\hline Rat FN & F: GACACTATGCGGGTCACTTG \\
R: CCCAGGCAGGAGATTTGTTA & F: TCATCGAATCGCACCTGAT \\
Rat IFN- $\gamma$ & R: GGATCTGTGGGTTGTTCACC \\
Fat IL-1 $\beta$ & F: GCCAACAAGTGGTATTCTCCA \\
& R: TGCCGTCTTTCATCACACAG \\
Rat IL-6 & F: GTCAACTCCATCTGCCCTTC \\
& R: TGTGGGTGGTATCCTCTGTG \\
Rat TNF- $\alpha$ & F: GCTCCCTCTCATCAGTTCCA \\
R: GCTTGGTGGTTTGCTACGAC & F: TGTTCACAGTTGCTGCCTGT \\
Rat MCP-1 & R: AGTTCTCCAGCCGACTCATT \\
Fat TGF- $\beta$ & F: CCAACTACTGCTTCAGCTCCA \\
& R: GTGTCCAGGCTCCAAATGT \\
Rat IL-8 & F: CTTTCAGAGACAGCAGAG \\
Rat $\beta-$-actin & F: CACAGTTCTTTAGCACTCC \\
& R: CCCATACCCACCATCACACC \\
&
\end{tabular}

FN, fibronectin; IFN- $\gamma$, interferon- $\gamma$; TNF- $\alpha$, tumor necrosis factor- $\alpha$; IL-1 $\beta$, interleukin-1 $\beta ; \mathrm{F}$, forward; R, reverse.

$0.9 \%$ saline, the CM-DiI-labeled MSCs were harvested in $0.9 \%$ saline. Approximately $5 \times 10^{6} \mathrm{MSCs}\left(1 \times 10^{7} / \mathrm{ml}, 0.5 \mathrm{ml}\right) / \mathrm{rat}$ were injected via the tail vein of 4 normal and 4 diabetic rats respecitvely, at 8 weeks after STZ injection. At 24 and $48 \mathrm{~h}$ after infusion of MSCs, rats were sacrificed and their kidney, thymus, spleen, lung, heart, liver and lymph node tissues were collected and processed to frozen sections. The labeled cells were observed under confocal microscopy (Nikon A1S1; Nikon, Tokyo, Japan).
Physical and biochemical analyses. The urine and serum samples were collected and renal function was monitored before treatment and at 3, 4, 6 and 8 weeks after initial MSC treatment. A total of 30 rats in three groups [(i) normal control group ( $\mathrm{NC} ; \mathrm{n}=5)$; (ii) DN group $(\mathrm{n}=14)$; (iii) DN-MSC group $(n=11)]$ were kept in metabolic cages to collect $24 \mathrm{~h}$ urine and serum samples that were obtained from the tail vein with a $26-G$ venous in-dwelling needle. Serum and urine samples were then centrifuged at $800 \times \mathrm{g}$ for $10 \mathrm{~min}$, and the supernatant was subjected to biochemical measurements including serum creatinine (Scr), blood urea nitrogen (BUN), glycosuria (GLU), microalbumin (MAU) and albumin to creatinine ratio (ACR). In addition, fasting blood glucose (FBG) of the rats was monitored each week. During the experiment, some of the rats, particularly those in the DN group were euthanized as certain humane endpoints were reached, such as inability to partake of food and water due to inability to move, or loss of appetite or severe diarrhea for $>3$ days, little or no reaction to the stimulation, etc.) At the end of the experiment, the surviving rats (14 rats in the DN group remained alive at the end of the experiment and 9 of the 11 rats in the MSCs group survived; rats in the normal control group all survived) were sacrificed and kidneys were harvested, weighed, and processed for histopathological analysis (Fig. 1).

Renal histology and immunohistochemistry. Kidney sections were stained with hematoxylin and eosin (H\&E), periodic acid-Schiff (PAS), and masson trichrome (MT; Richard-Allan Scientific, Kalamazoo, MI, USA) to assess the renal pathology. For immunohistochemical staining, rabbit anti-rat MCP-1 (ab25124; Abcam, Cambridge, MA, USA), IL-1 $\beta$ (ab82558; Abcam), IL-6 (ab6672; Abcam), mouse anti-rat TNF- $\alpha$ (ab1793; Abcam), ICAM-1 (ab2213; Abcam) and CD68 (ab74704; Abcam), transforming growth factor- $\beta$ (TGF- $\beta$; BS1361; Bioworld Technology, Inc., St. Louis Park, MN, USA), and fibronectin (FN; ab23751; Abcam) were used as the primary antibodies and biotinylated goat anti-rabbit or mouse IgG as the secondary antibody (Dako, Glostrup, Denmark). Standard immunohistochemical procedures were then used (28). The expression levels of the above factors were quantified by Image 
A

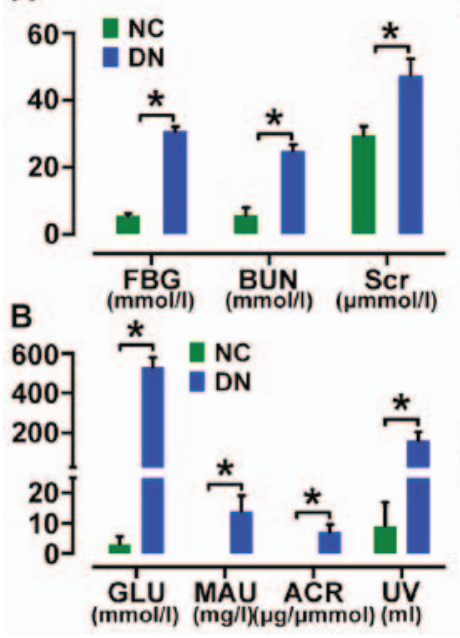

C NC DN

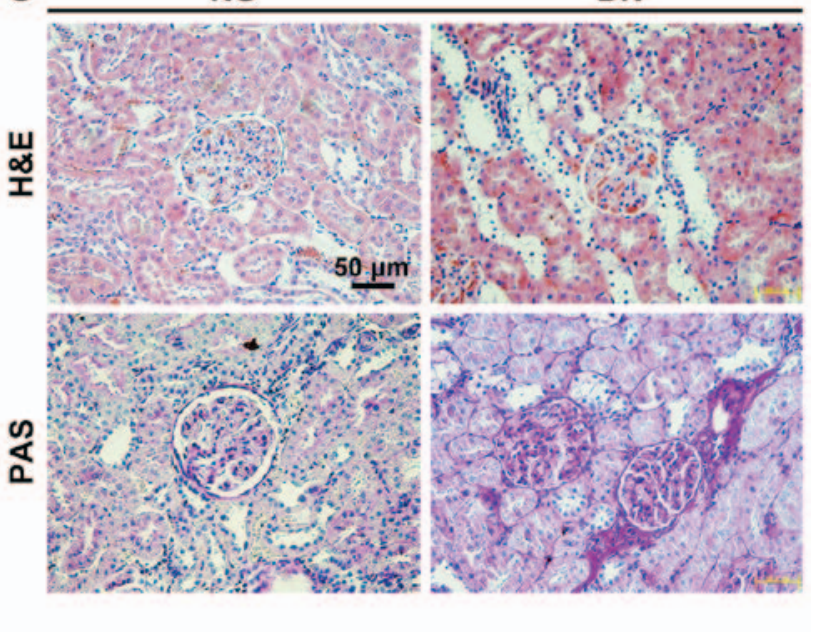

Figure 2. Assessment of renal injury in diabetic rats. Diabetic rats showed a significant increase in fasting blood glucose (FBG), serum creatinine (Scr), blood urea nitrogen (BUN) (A) as well as glycosuria (GLU), microalbumin (MAU), albumin to creatinine ratio (ACR) and urine volume (UV) (B) at 4 weeks after streptozotocin (STZ) injection. Hematoxylin and eosin (H\&E) and periodicacid-Schiff (PAS)-stained sections of renal cortices at 8 weeks (C) after diabetes onset showed obvious morphologic lesions in kidney of diabetic rats, including glomerular hypertrophy, increased fractional mesangial area and tubular dilatation [(C) magnification, $\mathrm{x} 400]\left[{ }^{*} \mathrm{p}<0.05\right.$ vs. normal group $\left.(\mathrm{NC})\right]$.

Pro-Plus v.6.0 software via analyzing the integral optical density in 10 non-overlapping cortical fields (magnification, $\mathrm{x} 400$ ).

Quantitative reverse transcription-PCR. Total RNA was extracted from renal cortical tissue or macrophage cells with TRIzol reagent (Invitrogen) according to the manufacturer's instructions, and then reverse transcribed into cDNA using Transcriptor First Strand cDNA synthesis kit (Roche, Indianapolis, IN, USA) and subjected to quantitative PCR using the iQ SYBR-Green Supermix with the iCycleriQ real-time PCR detection system (both from Bio-Rad Laboratories, Hercules, CA, USA). All the primers were designed and generated by Shenggong Biotechnology (Shanghai, China). Detailed primer information is presented in Table I. Each sample was tested in triplicates. The relative mRNA expression was determined as $2^{-\triangle \Delta C T}$ and normalized to the controls.

Serum cytokine expression. A 9-Plex rat cytokine/chemokine magnetic bead panel assay (Millipore Corp., St. Charles, MO, USA) was used to determine the cytokines in serum samples, including IL-1 $\alpha$, IL-1 $\beta$, IL-2, IL-6, epidermal growth factor (EGF), IL-10, TNF- $\alpha$, interferon- $\gamma$ (IFN- $\gamma$ ), GRO and VEGF. The samples were 1:2 diluted in the serum matrix. Beads were read on the Luminex 200 system (Luminex Corp., Austin, TX, USA) following the protocol and data were analyzed using Exponent 3.1 software.

Co-culture of MSCs and lipopolysaccharide (LPS)-stimulated macrophages. The isolated rat peritoneal macrophages were seeded intoa 24-well plate with or without 14-mm-diameter plastic coverslips at $1.5 \times 10^{5}$ cells/well. Two hours later, the medium was removed and the attached macrophages were co-cultured with $3 \times 10^{4}$ MSCs for $6 \mathrm{~h}$ with or without the stimulation of $100 \mathrm{ng} / \mathrm{ml}$ LPS. Afterward, cells were collected and the mRNA expression of inflammatory cytokines: MCP-1, IL-1 $\beta$, TNF- $\alpha$ and IL-6 were detected by qPCR. For immunofluorescence staining, coverslips with cells were fixed with $4 \%$ formaldehyde at room tempera- ture for $15 \mathrm{~min}$ and then permeabilized with $1 \%$ Triton X-100 in Tris buffer (Gibco) for another 15 min. Rabbit anti-rat MCP-1 (ab25124) and IL-6 (ab6672) (both from Abcam) were used as the primary antibodies and goat anti-rabbit $\mathrm{IgG}$ conjugated with FITC as the secondary antibody. To visualize nuclei, the cells were counter-stained with 4',6-diamidino-2-phenylindole (DAPI; Sigma Chemical) for $15 \mathrm{~min}$ at room temperature. The slides were examined under inverted fluorescence microscopy (Leica DM4000B; Leica, Wetzlar, Germany).

Statistical analysis. All values are presented as the means \pm SD. Statistical analysis was performed using SPSS 17.0 statistical software (SPSS Inc., Chicago, IL, USA). The statistical significance was analyzed by one-way ANOVA method with Turkey multiple comparison test. For survival experiments, a log-rank test was conducted. A level of $\mathrm{p}<0.05$ was considered statistically significant.

\section{Results}

Assessment of renal injury in a DN rat model. Rats with fasting glucose level $>16.7 \mathrm{mM}$ were considered diabetic. Urine analyses revealed the onset of microalbuminuria 2 weeks after STZ induction. Diabetic rats then showed a significant increase in serum levels of FBG, Scr and BUN, as well as MAU and ACR in urine (Fig. 2A and B) compared to those in age-matched control rats at 4 weeks after STZ injection. H\&E and PAS staining showed renal morphologic abnormalities in DN rats, including renal glomerular hypertrophy, increased collagen fibers, expansion of the mesangium, tubular dilatation and protein cylinders (Fig. 2C) at 8 weeks after STZ injection, confirming diabetic renal injury.

Characterization of rat MSCs. Bone marrow-derived MSCs from SD rats exhibited a typical fibroblast-like morphology (Fig. 3A) and were capable of differentiating into adipocytes after 14 days of culture and osteocytes after 21 days of culture (Fig. 3B), using histochemical staining for 
A
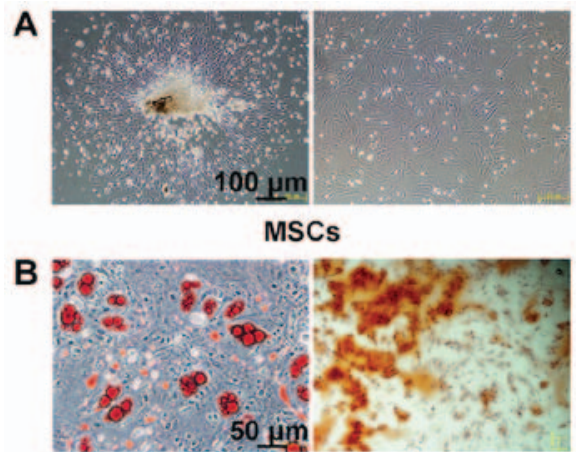

Oil red $\mathrm{O}$ staining Alizarin red staining

D

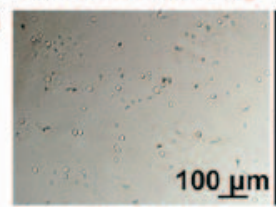

Bright field

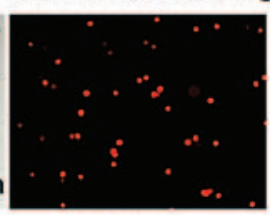

CM-Dil

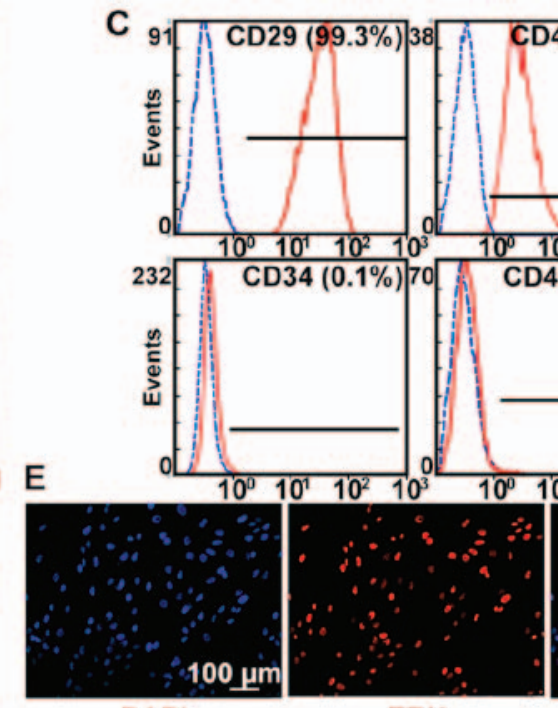

EDU

CD44 (99\%)

DAPI

Figure 3. Characterization of bone marrow-derived mesenchymal stem cells (MSCs) isolated from SD rats. Plastic adherent cells were ex vivo expanded [(A) magnification, x200] and differentiated into adipocytes after 14 days of culture or osteocytes after 21 days of culture [(B) magnification, x400]. Flow cytometry showed that MSCs at passage 3 were positive for CD29 (99.9\%), CD44 (99\%), and negative for CD34 (0.1\%) and CD45 (0.7\%) (C). MSCs were ex vivo labeled with CM-Dil [(D) magnification, $\mathrm{x} 200]$ and showed high labeling efficiency of almost $100 \%$. MSCs cultured with EdU ex vivo for $2 \mathrm{~h}$ showed $>60 \%$ positive staining indicating a high proliferative activity [(E) magnification, $\mathrm{x} 200]$.

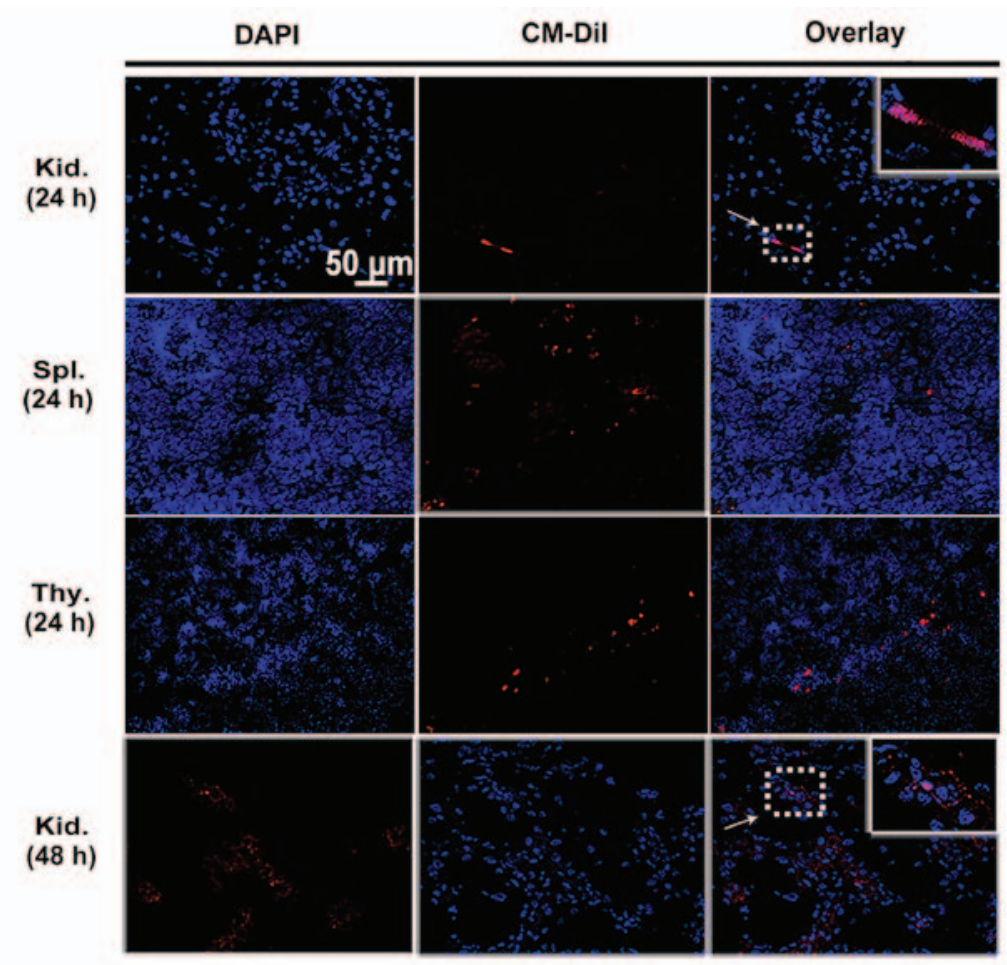

Figure 4. In vivo tracking of engrafted mesenchymal stem cells (MSCs) at 24 and $48 \mathrm{~h}$. Representative images of kidney (Kid.), spleen (Spl.) and thymus (Thy.) sections from diabetic rats injected with $5 \times 10^{6}$ of CM-DiI labeled MSCs at 24 and $48 \mathrm{~h}$ after cell infusion. MSCs labeled with CM-DiI showed red fluorescence (arrow), and nuclei were stained by DAPI with blue fluorescence. Magnification, x400.

lineage-specific markers. Flow cytometry showed that MSCs at passage 3 were positive for $\mathrm{CD} 29(99.3 \%)$ and CD44 (99\%), and negative for $\mathrm{CD} 34(0.1 \%)$ and $\mathrm{CD} 45$ (0.7\%) (Fig. 3C). MSCs labeled by CM-DiI showed red fluorescence, and the labeling efficiency was almost 100\% (Fig. 3D). In addition, MSCs cultured with EdU ex vivo for $2 \mathrm{~h}$ showed more than $60 \%$ positive staining, indicating a high proliferative activity (Fig. 3E).
Intravenously injected MSCs localize in the kidney and immune organs of DN rats. MSCs were labeled with CM-DiI to track their homing after being transplanted to peripheral circulation of rats. Engrafted MSCs with red fluorescence were detected in the glomeruli as well as tubulointerstitium of diabetic kidneys by confocal microscopy at 24 and $48 \mathrm{~h}$ after cell infusion (Fig. 4). Cell engraftment was also observed in the 

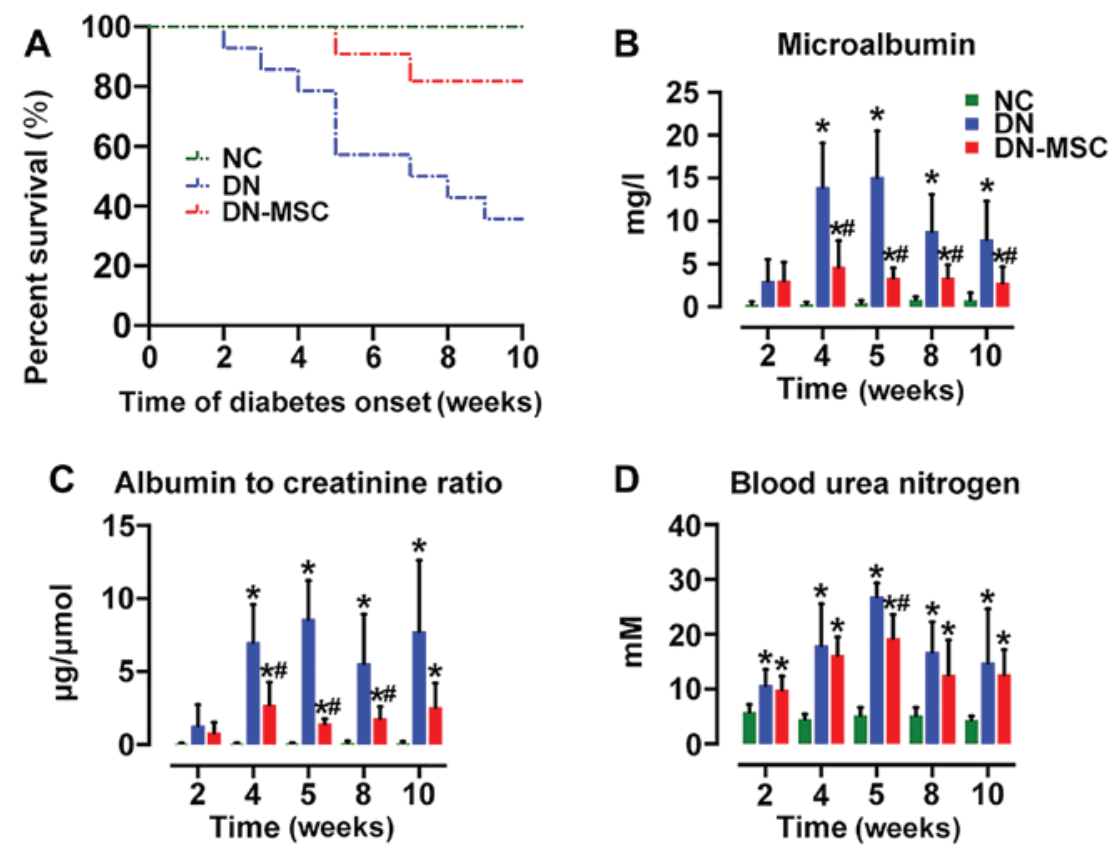

Figure 5. Effect of mesenchymal stem cells (MSCs) on survival rate, body weight and renal function. (A) Ten-week survival rates of the 3 groups: NC group, 100\%; diabetic nephropathy (DN) group, 35.7\%; DN-MSC group, 81.8\% ("p=0.01). Data were analyzed by log-rank test. Renal function: (B) microalbumin (MAU) (mg/l), (C) albumin to creatinine ratio (ACR) $(\mu \mathrm{g} / \mu \mathrm{mol})$ and (D) blood urea nitrogen (BUN) (mM) were measured in serum and urine in the 3 groups at 4, 5, 8 and 10 weeks after diabetes onset. Renal function was significantly improved in the MSC-treated group compared with that noted in the DN group. Data are expressed as means $\pm \mathrm{SD}$ of evaluations from each group. [" $\mathrm{p}<0.05$ vs. normal group (NC); ${ }^{\sharp} \mathrm{p}<0.05 \mathrm{vs.} \mathrm{DN} \mathrm{group].}$

immune organs such as spleen and thymus until $24 \mathrm{~h}$ (Fig. 4). Interestingly, more CM-DiI-labeled cells were found in the thymuses. However, the number of MSCs positive for CM-DiI declined over time. When MSCs were intravenously injected in normal control rats, no CM-DiI-labeled cells were found in the kidney or immune organs (data not shown).

MSCs extend the survival of DN rats and ameliorate renal function. At 10 weeks after diabetes onset, the survival of the DN group was markedly decreased to $35.7 \%$ (5/14) in comparison with $100 \%$ survival of the NC group. MSC transplantation significantly extended the survival of DN rats, exhibiting $81.8 \%$ survival (9/11) in the DN-MSC group (Fig. 5A). Although diabetic rats showed marked elevations in MAU, ACR and BUN, treatment with MSCs resulted in the suppression of STZ-induced detrimental impact on the above parameters at 4, 5, 8 and 10 weeks after diabetes onset (Fig. 5B-D). However, only a slight decrease in serum glucose level was observed in the DN-MSC rats in comparison with that in the DN animals.

MSCs preserve renal structure and inhibit fibrosis. Ten weeks after diabetes onset, H\&E staining of renal tissues from the DN rats showed typical chronic kidney injury, including glomerular hypertrophy, epithelial flattening and dilated tubules. Notable, histology of the DN-MSC rats was similar to that of the control group with normal glomerular and tubular morphology. In addition, kidneys of the DN group rats exhibited profound extracellular matrix deposition and frequent fibrin cap formation inside the glomeruli as revealed by PAS staining, whereas, distinct decrease in mesangial matrix deposition and glomerulosclerosis was observed in the MSC-treated group. Masson's trichrome staining also demonstrated a significant reduction in fibrosis in the DN-MSC rats (Fig. 6A). Meanwhile, the expression levels of TGF- $\beta$ and FN in kidney, which are typical biomarkers of epithelial-mesenchymal transition (EMT) and fibrosis, were substantially downregulated in the MSC-treated rats compared with DN rats as detected by immunohistochemistry (Fig. 6B and C).

Regulatory effect of MSCs on the immune microenvironment MSCs reduced renal CD68-positive macrophage infiltration and inflammatory cytokine expression. MCP-1, and ICAM-1 have been known as essential signals for macrophage activation and recruitment. In the present study, the immunostaining for MCP-1 and ICAM-1 was significantly increased in the DN group in glomeruli and interstitium compared with the non-diabetic rats, but markedly reduced by MSC treatment. CD68-positive cells infiltrated in the glomeruli of the diabetic rats at 10 weeks after STZ injection. But MSC treatment markedly suppressed the macrophage infiltration into the glomeruli (Fig. 7A and B). Consistently, the markedly induced expression of pro-inflammatory cytokines TNF- $\alpha$, IL- 6 and IL-1 $\beta$ in the kidneys of DN rats was abrogated by MSC treatment in the DN-MSC group (Fig. 7C and D).

MSCs decrease renal inflammatory gene expression. Diabetic rats exhibited increased gene expression of proinflammatory cytokines TNF- $\alpha$, IL-6, IL-8, IL- $1 \beta$ and IFN- $\gamma$, chemokine MCP-1, and fibrosis markers TGF- $\beta$ and FN compared with the non-diabetic animals. However, treatment with MSCs resulted in the notable suppression in gene expression of the above factors 10 weeks after diabetes onset (Fig. 8).

MSCs suppress serum inflammatory cytokines and enhance anti-inflammatory cytokines. In regards to the immunosuppressive properties of MSCs, we measured the 

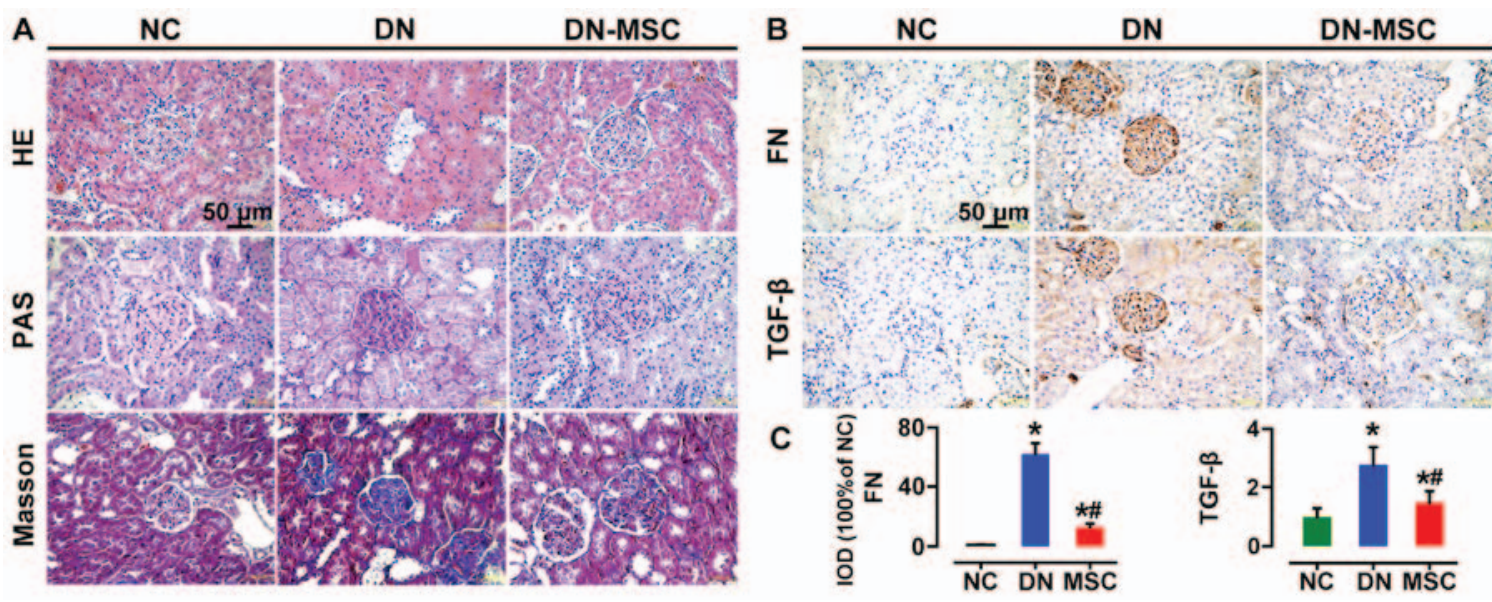

Figure 6. Effect of mesenchymal stem cells (MSCs) on renal histopathological changes and expression of fibronectin (FN) and transforming growth factor- $\beta$ (TGF- $\beta$ ) at 10 weeks after diabetes onset. (A) Representative images of hematoxylin and eosin (H\&E), periodic acid-Schiff (PAS) and trichromestained sections of renal cortices from three groups of rats. Severe histologic changes were visible in the diabetic nephropathy (DN) group, including glomerular hypertrophy, increased fractional mesangial area and interstitial fibrosis. Recovery from most of the glomerular and tubular changes were observed in the MSC-treated group rats. (B) Immunohistochemical analyses of FN and TGF- $\beta$ protein expression in kidney tissues of the three groups of rats. (C) Quantitative analyses of FN and TGF- $\beta$ expression as measured by immunohistochemistry (IHC) (100\% of normal control). Data are expressed as means \pm SD of evaluations from each group [ ${ }^{*} \mathrm{p}<0.05$ vs. normal group $(\mathrm{NC}) ;{ }^{\#} \mathrm{p}<0.05$ vs. DN group]. Magnification, $\mathrm{x} 400$.
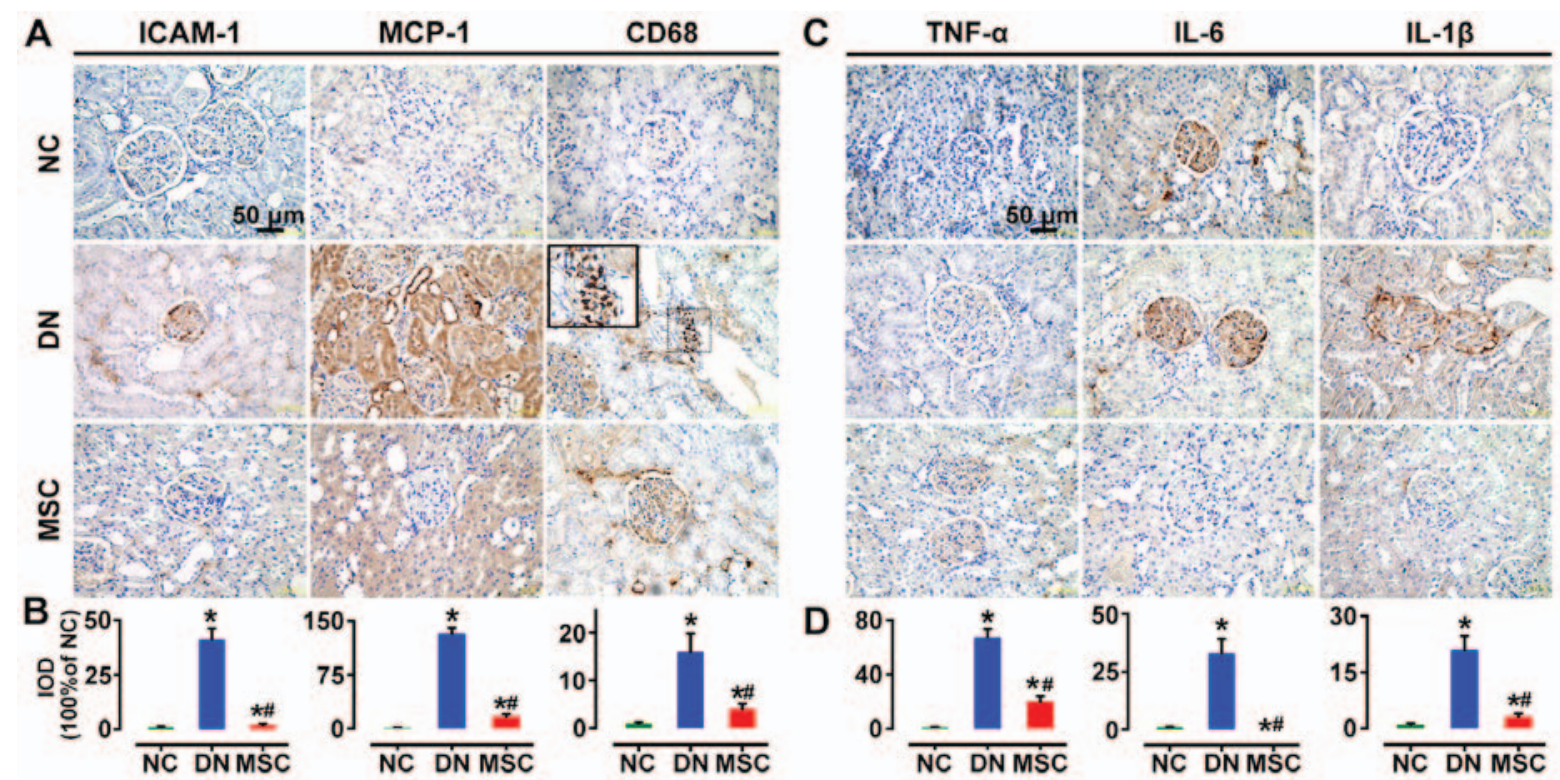

Figure 7. Effect of mesenchymal stem cells (MSCs) on renal macrophage infiltration and pro-inflammatory cytokine expression 10 weeks after diabetes onset. (A) Immunohistochemistry (IHC) analyses of ICAM-1, MCP-1 and CD68 protein expression in kidney tissues of the three groups of rats. (B) Quantitative analyses of ICAM-1, MCP-1 and CD68 expression as measured by IHC. The number of CD68 ${ }^{+}$macrophages was significantly suppressed after treatment with MSCs. (C) IHC analysis of TNF- $\alpha$, interleukin-6 (IL-6) and IL-1 $\beta$ protein expression in kidney tissues of three groups of rats. (D) Quantitative analyses of TNF- $\alpha$, IL-6 and IL-1 $\beta$ protein expressions as measured by IHC (100\% of normal control). Data are expressed as means \pm SD of evaluations from each group $\left[{ }^{*} \mathrm{p}<0.05\right.$ vs. normal group (NC); ${ }^{\#} \mathrm{p}<0.05$ vs. diabetic nephropathy (DN) group]. Magnification, $\mathrm{x} 400$.

serum levels of cytokines to further explore the impact of MSCs on the immune environment of DN 10 weeks after diabetes onset. Diabetic rats exhibited increased serum levels of IL- $1 \alpha$, IL-1 $\beta$, IL-6, IFN- $\gamma$ and GRO (CXCL1) in comparison with the non-diabetic control rats. Treatment with MSCs significantly attenuated the expression of IL-1 $\alpha$, IL-1 $\beta$, IL-6 and IFN- $\gamma$ (Fig. 9). Notably, the level of EGF was obviously higher in the MSC-treated rats than in both the control and diabetic rats. However, no obvious change was observed in serum levels of IL-10, VEGF and TNF- $\alpha$ after MSC treatment.
MSCs suppress pro-inflammatory cytokine profile in activated macrophages ex vivo. To further assess the effect of MSCs on macrophage activation, we examined the alteration of mRNA profiles of pro-inflammatory cytokines in LPS-stimulated rat peritoneal macrophages before and after MSC treatment. Macrophages were identified as having positive expression of CD68 (63.9\%) and F4/80 (51.2\%) by flow cytometry (Fig. 10A and B), markers of activated macrophages. Pro-inflammatory cytokines, such as IL-6, IL-1 $\beta$, TNF- $\alpha$ and MCP-1 were highly expressed in LPS-stimulated macrophages. However, their mRNA expression levels were 


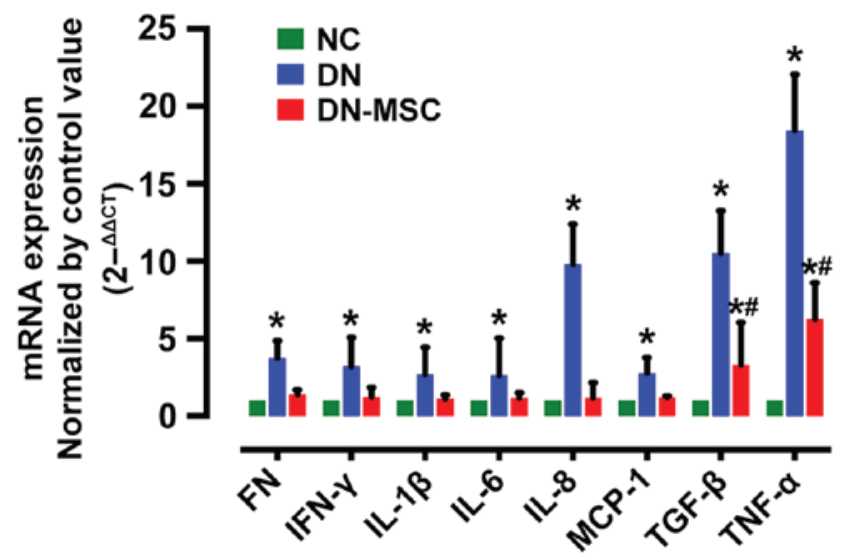

Figure. 8 Effect of mesenchymal stem cells (MSCs) on pro-inflammatory, fibrotic cytokine and chemokine gene expression in kidney tissues. The mRNA expression of cytokines and fibronectin (FN) were significantly suppressed in the MSC group compared with the diabetic nephropathy (DN) group at 10 weeks after diabetes onset. All the data were normalized by control value (control was shown as 1 ), and are presented as means $\pm \mathrm{SD}$ $\left[{ }^{*} \mathrm{p}<0.05\right.$ vs. normal group (NC); ${ }^{\#} \mathrm{p}<0.05$ vs. DN group].

significantly downregulated following co-culture with MSCs (Fig. 10C). In addition, immunofluorescence showed consistent results. LPS-induced MCP-1 and IL-6 expression was decreased by MSC intervention (Fig. 10D). These results demonstrated that co-culturing with MSCs led to inhibition of macrophage activation, which was relevant to the immunomodulatory characteristics of MSCs.

\section{Discussion}

To date, diabetic nephropathy (DN) is still incurable and is a big challenge for clinical practice. Previous experimental studies of DN suggest that inflammation contributes to its pathogenesis (9). Increasing evidence has revealed that interactions of MSCs with macrophages are likely to play a significant role in their anti-inflammatory/immune modulatory effects (29), which may be a critical mechanism in MSC-mediated amelioration of inflammation-related diseases (24). In the present study, we found that the early intervention of diabetes by MSCs preserved renal function and ameliorated histopathological alterations in a rat model of diabetes mellitus. The possible underlying mechanisms are suggested to be the inhibition of macrophage activation and the trigger for a pro-regenerative microenvironment, including the production of protective trophic factors and the attenuation of the pro-inflammatory response in the kidney, resulting in improved renal dysfunction.

STZ-induced rat type 1 diabetes was used in this study to mimic clinical $\mathrm{DN}$, as it is a well documented type 1 diabetic model to quickly and stably develop hyperglycemia and renal injury (30). We observed that 8 weeks after STZ injection, diabetic rats were severely hyperglycemic and developed typical alterations of DN. Serum MAU, Scr and urine ACR were increased, as well as significant pathological lesions were observed, including glomerular, tubular epithelial hypertrophy, profound extracellular matrix deposition and fibrosis, suggesting successful establishment of the DN rat model (31) (Fig. 2). In terms of the timing of MSC intervention, previous studies mostly conducted MSC infu-

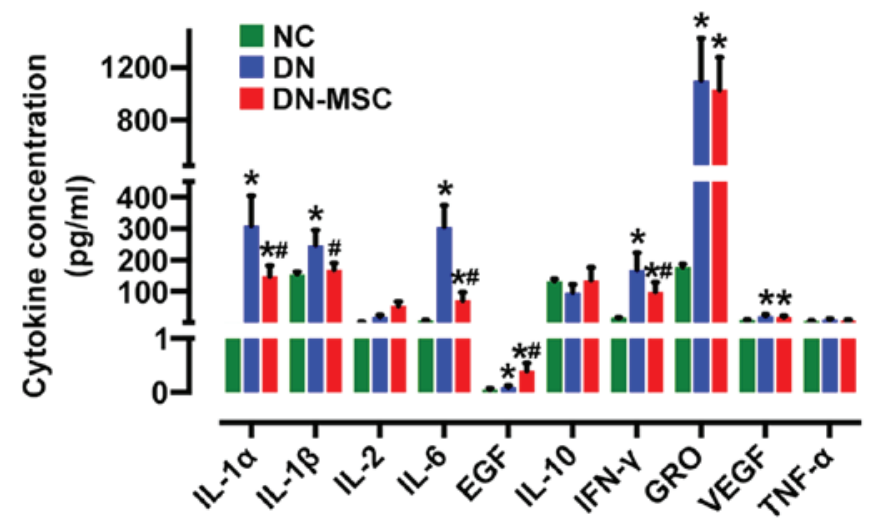

Figure 9. Effect of mesenchymal stem cells (MSCs) on the serum inflammatory cytokines and growth factor expression. The levels of cytokines in the serum samples were determined by a Milliplex rat cytokine kit in the three groups of rats at 10 weeks after diabetes onset. Interleukin-1 $\alpha$ (IL-1 $\alpha$ ), IL-1 $\beta$, IL-6, interferon- $\gamma($ IFN $-\gamma)$ expression lelvels were significantly suppressed in the MSC group, whereas epidermal growth factor (EGF) was increased compared with the diabetic nephropathy (DN) group. Data are expressed as means \pm SD of evaluations from each group [ $\mathrm{p} p<0.05$ vs. normal group $(\mathrm{NC}) ;{ }^{\#} \mathrm{p}<0.05$ vs. DN group].

sion after the animals already established DN $(32,33)$. In this study, given that it is difficult to reverse the advanced stage of nephropathy, we chose 2 weeks after diabetes onset as the timing for MSC therapy, due to the fact that rats in the very early stage of DM are more sensitive to MSC intervention. As expected, our results showed a better preventive effect on renal injury than studies conducting MSC transplantation in severe DN (32,33). Most importantly, in this study, MSC-treated diabetic rats did not develop serious renal histopathologic alterations, suggesting that MSC administration hindered further renal impairment. However, previous studies only observed mild microalbuminuria reversion as well as amelioration of glomeruli structural damage. On the other hand, the diabetic rats received 4 consecutive MSC infusions in the present study, which was similar with the strategy reported by Semedo et al. They conducted a 6 -week MSC treatment of 3 consecutive infusions every other week, showing obvious amelioration of kidney function, which was better than single dose treatment (22). Parameters used for evaluation of renal function are variable in the literature. Although in many studies, functional parameters were only measured at the end of the experiment $(14,32)$, we monitored the dynamic changes of serum and urine biochemistry with the purpose of assessing diabetic status and progression of renal injury. We found that the administration of multiple doses of MSCs was capable of preventing albuminuria development regardless of the persistence of hyperglycemia at all time points. MSCs effectively restored renal function from 4 weeks after diabetes onset (2 weeks after MSC transplantation), strikingly improving MAU and ACR (Fig. 5). Notably, the above renal indices were increased in the DN group rats from 2 weeks after diabetes onset and peaked at 5 weeks. Afterwards, BUN and MAU in the DN group displayed a gradual decline. This may be due to the auto-repair potential of kidneys after high glucose damage.

Previous studies have also shown that the development of diabetes leads to increased innate immune responses, which are predominantly characterized by the accumulation of kidney macrophages (34). Activated macrophages secrete a series 
A

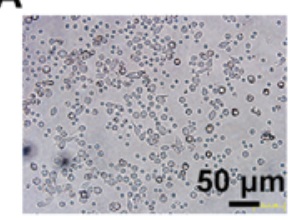

C
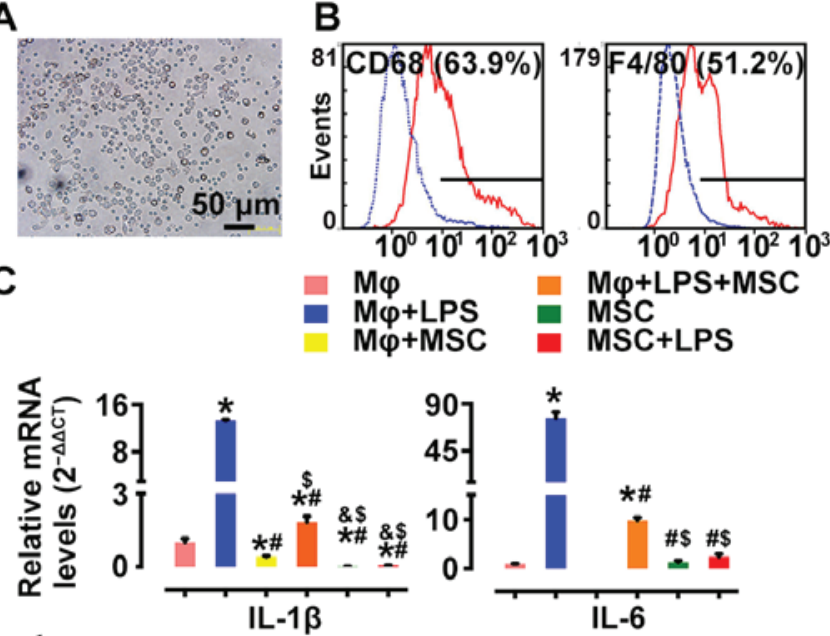

$M \varphi+L P S+M S C$

MSC

MSC+LPS
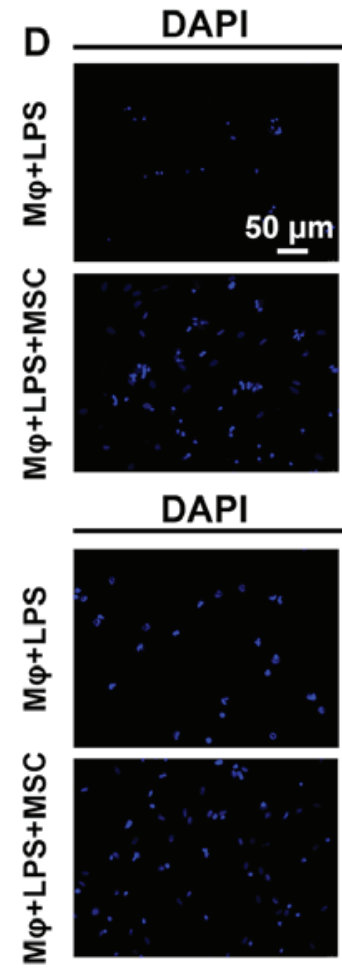

MCP-1
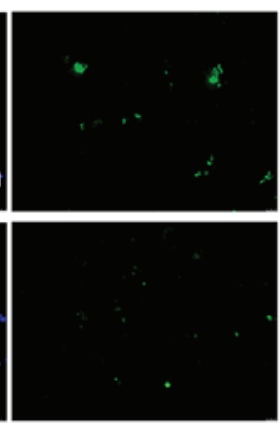

IL-6
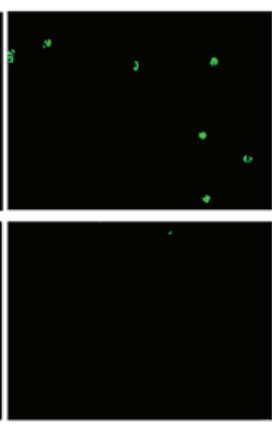

Overlay
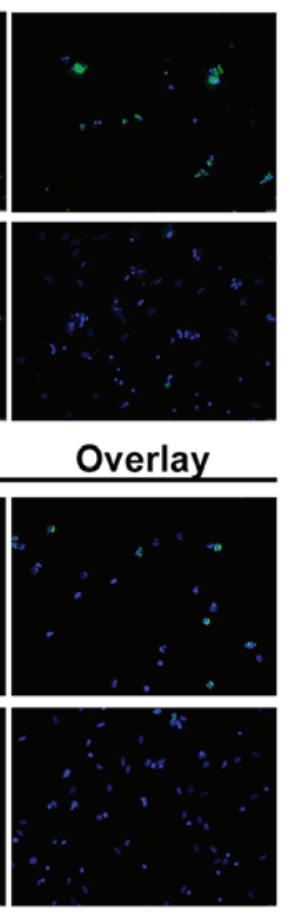

Figure 10. Effect of mesenchymal stem cells (MSCs) on macrophage activation stimulated by lipopolysacharide (LPS). Primarily isolated rat peritoneal macrophages $\mathrm{M} \phi$ (bright field) (A) were identified as being positive for (B) CD68 (63.9\%) and F4/80 (51.2\%) by flow cytometry. qPCR analyses of (C) interleukin-6 (IL-6), MCP-1, IL-1 $\beta$, TNF- $\alpha$ and cellular immunofluorescence staining of IL-6 and MCP-1 [(D) magnification, x200] showed that expression of inflammatory mediators secreted by LPS-stimulated macrophages were inhibited by co-culturing with MSCs at $6 \mathrm{~h}$. Data are expressed as means \pm SD of evaluations from each group ( ${ }^{*} \mathrm{p}<0.05$ vs. M $\phi ;{ }^{*} \mathrm{p}<0.05$ vs. M $\phi+\mathrm{LPS} ;{ }^{\&} \mathrm{p}<0.05$ vs. M $\phi+\mathrm{MSC} ;{ }^{\$} \mathrm{p}<0.05$ vs. M $\left.\phi+\mathrm{LPS}+\mathrm{MSC}\right)$.

of pro-inflammatory, pro-fibrogenic and anti-angiogenesis cytokines, which are responsible for a pro-inflammatory microenvironment (35) and their infiltrations are strongly associated with proteinuria and declined renal function $(10,13)$. In addition, this finding was supported by in vitro studies indicating that activated macrophages can promote renal cell death and stimulation of bone marrow-derived macrophages by LPS resulting in the secretion of substances known to induce apoptosis in cultured mesangial and tubular epithelial cells (36). In addition, kidney cells produce ICAM-1 and MCP-1 in response to a variety of pro-inflammatory stimuli (9). In patients with DN, soluble forms of ICAM are elevated during the progression of the disease and experimental studies suggest that ICAM-1 facilitates kidney macrophage recruitment during type 1 diabetes (37). Furthermore, MCP-1 is a specific chemokine to recruit and activate monocytes from the circulation to the inflammatory site (38), especially contributing to infiltration of macrophages into both mesangial and tubulointerstitial lesions in DN (39). In the present study, treatment with MSCs markedly attenuated MCP-1 and ICAM-1 expression, and diminished the accumulation of macrophages in glomeruli (Figs. 7 and 8), which is consistent with previous studies $(40,41)$. Additionally, expression levels of classical markers of activated macrophages, such as IL- $1 \beta$, TNF- $\alpha$ and IL- 6 as well as fibrosis factors TGF- $\beta$ and FN in the MSC group were significantly declined at the mRNA and/or protein levels (Figs. 7 and 8), which implied that the inflammatory environment of DN was substantially improved by MSCs. Furthermore, our ex vivo study also provided more direct evidence that MSCs were able to diminish the LPS driven activation of macrophages (Fig. 10).
Summarily, these results suggested that the anti-inflammatory action of MSCs in the DN rat model was related to the modulation of macrophages. Recently, it has been shown that MSC administration reduces macrophage infiltration into the target tissues (29) and switches macrophages from an M1 to M2 phenotype, benefiting tissue homeostasis in different animal models (42). The in-depth study of the mechanism involved in the MSC-mediated modulation of macrophages in DN is warranted for better understanding of its therapeutic effects.

It is undoubted that MSCs have the capability to modulate the local inflammatory environment, and perturb local interactions with inflammatory cells in diabetic kidney. We aimed to ascertain whether MSCs alter systemic inflammation in this scenario. The evaluation of specific cytokines in the serum demonstrated that MSC administration led to a reduction in serum pro-inflammatory cytokines, including IL-6, IL-1 $\alpha$, IL-1 $\beta$ and IFN- $\gamma$. Notably, we also observed a systemic increase in EGF and IL-10 (Fig. 8). This immunosuppressive property of MSCs has been described in a great number of literature studies, including inhibition of the proliferation of $\mathrm{CD}^{+}$and $\mathrm{CD}^{+} \mathrm{T}$ lymphocytes and natural killer $(\mathrm{NK})$ cells, inhibition of maturation of dendritic cells (DCs), and stimulation of the proliferation of regulatory T cells (16). This immune modulation is thought to be due to the paracrine action of soluble factors. The secretion of prostaglandin $\mathrm{E}_{2}\left(\mathrm{PGE}_{2}\right)$, inducible nitric oxide synthase (iNOS), indoleamine-2,3-dioxygenase (IDO), TGF- $\beta$, leukemia-inhibitory factor (LIF), and IL-10 contributes to this effect (15). Moreover, trophic factors, such as VEGF, HGF, IGF-1 and EGF have been shown to reduce tubular injury or mediate epithelial cell proliferation (43). 
To fully understand the mechanism underlying the effect of MSCs, how MSCs are localized in deteriorated kidneys must be determined (Fig. 4). Although the exact mechanism involved in stem cell homing still remains elusive, inflammation, hypoxia, and hyperglycemia, which are ongoing in the diabetic kidney, may be the driving factors of MSC migration (44-46). In addition, MSCs may be able to both sense and respond to their immediate environment, which makes them ideal cells to tune the response to injury and inflammation (15). Notably, cell engraftments were found in the immune organs such as thymus and spleen (Fig. 4). Previous studies have shown that activated immunocytes secrete IFN- $\gamma$, promoting neighboring MSCs to secrete $\mathrm{PGE}_{2}$, which could regulate the functions of a variety of immune cells (47). Meanwhile, it has been shown that inhibition of allogeneic $\mathrm{T}$ cell responses by MSCs is mediated by indoleamine-2,3-dioxygenase (IDO) (48). IDO is responsible for catabolizing tryptophan through the kyneurinine pathway, thus limiting the availability of L-tryptophan to $\mathrm{T}$ cells resulting in nutrition deficiency-induced cell death (49). Taken together, this implies that the obvious tendency of MSCs to engraft in immune organs is also a determining factor for the MSC-mediated immunosuppressive effects and alleviation of inflammation in the damaged kidney via systemic immunoregulation.

In conclusion, we presented evidence that the early intervention by MSCs effectively suppressed renal macrophage infiltration and inflammatory cytokine expression in diabetic rats via immunoregulation and paracrine actions, which restored the homeostasis of the immune microenvironment and led to the amelioration of kidney function and glomerulosclerosis. In addition, MSCs infused via the tail vein were able to home to deteriorated kidney and immune organs. Ex vivo, MSCs inhibited activation of rat peritoneal macrophages after LPS stimulation. We suggest that the prevention of DN with MSCs may provide substantial promise for the development of novel MSC-based interventions.

\section{Acknowledgements}

This study was supported by the National Natural Science Foundation of China (no. 81370824) and the National Key Clinical Project.

\section{Competing interests}

The authors declare that they have no competing interests.

\section{References}

1. Dronavalli S, Duka I and Bakris GL: The pathogenesis of diabetic nephropathy. Nat Clin Pract Endocrinol Metab 4: 444-452, 2008.

2. Maisonneuve P, Agodoa L, Gellert R, Stewart JH, Buccianti G, Lowenfels AB, Wolfe RA, Jones E, Disney APS, Briggs D, et al: Distribution of primary renal diseases leading to end-stage renal failure in the United States, Europe, and Australia/New Zealand: Results from an international comparative study. Am J Kidney Dis 35: 157-165, 2000.

3. Chavers BM, Bilous RW, Ellis EN, Steffes MW and Mauer SM: Glomerular lesions and urinary albumin excretion in type I diabetes without overt proteinuria. N Engl J Med 320: 966-970, 1989.

4. McCrary EB: The road to renal failure: An overview of diabetic nephropathy. Adv Nurse Pract 16: 61-63, 2008.

5. Schena FP and Gesualdo L: Pathogenetic mechanisms of diabetic nephropathy. J Am Soc Nephrol 16 (Suppl 1): S30-S33, 2005.
6. Navarro-González JF, Mora-Fernández C, Muros de Fuentes M and García-Pérez J: Inflammatory molecules and pathways in the pathogenesis of diabetic nephropathy. Nat Rev Nephrol 7: 327-340, 2011.

7. Navarro-González JF and Mora-Fernández C: The role of inflammatory cytokines in diabetic nephropathy. J Am Soc Nephrol 19: 433-442, 2008

8. Cho DI, Kim MR, Jeong HY, Jeong HC, Jeong MH, Yoon SH, Kim YS and Ahn Y: Mesenchymal stem cells reciprocally regulate the $\mathrm{M} 1 / \mathrm{M} 2$ balance in mouse bone marrow-derived macrophages. Exp Mol Med 46: e70, 2014.

9. Nguyen D, Ping F, Mu W, Hill P, Atkins RC and Chadban SJ: Macrophage accumulation in human progressive diabetic nephropathy. Nephrology (Carlton) 11: 226-231, 2006.

10. Chow F, Ozols E, Nikolic-Paterson DJ, Atkins RC and Tesch GH: Macrophages in mouse type 2 diabetic nephropathy: Correlation with diabetic state and progressive renal injury. Kidney Int 65: 116-128, 2004.

11. Cao Q, Wang Y, Zheng D, Sun Y, Wang Y, Lee VWS, Zheng G, Tan TK, Ince J, Alexander SI, et al: IL-10/TGF- $\beta$-modified macrophages induce regulatory $\mathrm{T}$ cells and protect against adriamycin nephrosis. J Am Soc Nephrol 21: 933-942, 2010.

12. Prockop DJ and Oh JY: Mesenchymal stem/stromal cells (MSCs): Role as guardians of inflammation. Mol Ther 20: 14-20, 2012.

13. Chow FY, Nikolic-Paterson DJ, Atkins RC and Tesch GH: Macrophages in streptozotocin-induced diabetic nephropathy: potential role in renal fibrosis. Nephrol Dial Transplant 19: 2987-2996, 2004.

14. Wang S, Li Y, Zhao J, Zhang J and Huang Y: Mesenchymal stem cells ameliorate podocyte injury and proteinuria in a type 1 diabetic nephropathy rat model. Biol Blood Marrow Transplant 19: 538-546, 2013.

15. Singer NG and Caplan AI: Mesenchymal stem cells: Mechanisms of inflammation. Annu Rev Pathol 6: 457-478, 2011.

16. Volarevic V, Arsenijevic N, Lukic ML and Stojkovic M: Concise review: Mesenchymal stem cell treatment of the complications of diabetes mellitus. Stem Cells 29: 5-10, 2011.

17. Lee RH, Seo MJ, Reger RL, Spees JL, Pulin AA, Olson SD and Prockop DJ: Multipotent stromal cells from human marrow home to and promote repair of pancreatic islets and renal glomeruli in diabetic NOD/scid mice. Proc Natl Acad Sci USA 103: 17438-17443, 2006.

18. Tögel F, Hu Z, Weiss K, Isaac J, Lange C and Westenfelder C: Administered mesenchymal stem cells protect against ischemic acute renal failure through differentiation-independent mechanisms. Am J Physiol Renal Physiol 289: F31-F42, 2005.

19. Tögel F, Weiss K, Yang Y, Hu Z, Zhang P and Westenfelder C: Vasculotropic, paracrine actions of infused mesenchymal stem cells are important to the recovery from acute kidney injury. Am J Physiol Renal Physiol 292: F1626-F1635, 2007.

20. Ezquer FE, Ezquer ME, Parrau DB, Carpio D, Yañez AJ and Conget PA: Systemic administration of multipotent mesenchymal stromal cells reverts hyperglycemia and prevents nephropathy in type 1 diabetic mice. Biol Blood Marrow Transplant 14: 631-640, 2008.

21. Semedo P, Palasio CG, Oliveira CD, Feitoza CQ, Gonçalves GM, Cenedeze MA, Wang PMH, Teixeira VPA, Reis MA, Pacheco-Silva A, et al: Early modulation of inflammation by mesenchymal stem cell after acute kidney injury. Int Immunopharmacol 9: 677-682, 2009.

22. Semedo P, Correa-Costa M, Antonio Cenedeze M, Maria Avancini Costa Malheiros D, Antonia dos Reis M, Shimizu MH, Seguro AC, Pacheco-Silva A, Saraiva Camara NO and Olsen N: Mesenchymal stem cells attenuate renal fibrosis through immune modulation and remodeling properties in a rat remnant kidney model. Stem Cells 27: 3063-3073, 2009.

23. Park JH, Hwang I, Hwang SH, Han H and Ha H: Human umbilical cord blood-derived mesenchymal stem cells prevent diabetic renal injury through paracrine action. Diabetes Res Clin Pract 98: 465-473, 2012.

24. Nakajima H, Uchida K, Guerrero AR, Watanabe S, Sugita D, Takeura N, Yoshida A, Long G, Wright KT, Johnson WEB, et al: Transplantation of mesenchymal stem cells promotes an alternative pathway of macrophage activation and functional recovery after spinal cord injury. J Neurotrauma 29: 1614-1625, 2012.

25. Jiang TS, Cai L, Ji WY, Hui YN, Wang YS, Hu D and Zhu J: Reconstruction of the corneal epithelium with induced marrow mesenchymal stem cells in rats. Mol Vis 16: 1304-1316, 2010. 
26. Mafi P, Hindocha S Mafi R, Griffin M and Khan WS: Adult mesenchymal stem cells and cell surface characterization - a systematic review of the literature. Open Orthop J 5 (Suppl 2): 253-260, 2011.

27. Zhang X, Goncalves R and Mosser DM: The isolation and characterization of murine macrophages. Curr Protoc Immunol: Chapter: Unit-14.1, 2008

28. Wang Y, Wang Y, Feng X, Bao S, Yi S, Kairaitis L, Tay YC, Rangan GK and Harris DC: Depletion of CD4(+) T cells aggravates glomerular and interstitial injury in murine adriamycin nephropathy. Kidney Int 59: 975-984, 2001.

29. Kim J and Hematti P: Mesenchymal stem cell-educated macrophages: A novel type of alternatively activated macrophages. Exp Hematol 37: 1445-1453, 2009.

30. Tesch GH and Allen TJ: Rodent models of streptozotocin-induced diabetic nephropathy. Nephrology (Carlton) 12: 261-266, 2007.

31. Liu CX, Hu Q, Wang Y, Zhang W, Ma ZY, Feng JB, Wang R, Wang XP, Dong B, Gao F, et al: Angiotensin-converting enzyme (ACE) 2 overexpression ameliorates glomerular injury in a rat model of diabetic nephropathy: A comparison with ACE inhibition. Mol Med 17: 59-69, 2011.

32. Lv SS, Liu G, Wang JP, Wang WW, Cheng J, Sun AL, Liu HY, Nie HB, Su MR and Guan GJ: Mesenchymal stem cells transplantation ameliorates glomerular injury in streptozotocin-induced diabetic nephropathy in rats via inhibiting macrophage infiltration. Int Immunopharmacol 17: 275-282, 2013.

33. Lv S, Cheng J, Sun A, Li J, Wang W, Guan G, Liu G and Su M: Mesenchymal stem cells transplantation ameliorates glomerular injury in streptozotocin-induced diabetic nephropathy in rats via inhibiting oxidative stress. Diabetes Res Clin Pract 104: 143-154, 2014.

34. Tesch GH: MCP-1/CCL2: A new diagnostic marker and therapeutic target for progressive renal injury in diabetic nephropathy. Am J Physiol Renal Physiol 294: F697-F701, 2008.

35. Brady HR: Leukocyte adhesion molecules and kidney diseases. Kidney Int 45: 1285-1300, 1994.

36. Duffield JS, Erwig L-P, Wei X, Liew FY, Rees AJ and Savill JS: Activated macrophages direct apoptosis and suppress mitosis of mesangial cells. J Immunol 164: 2110-2119, 2000.

37. Chow FY, Nikolic-Paterson DJ, Ozols E, Atkins RC and Tesch GH: Intercellular adhesion molecule-1 deficiency is protective against nephropathy in type 2 diabetic $\mathrm{db} / \mathrm{db}$ mice. J Am Soc Nephrol 16: 1711-1722, 2005.

38. Banba N, Nakamura T, Matsumura M, Kuroda H, Hattori Y and Kasai K: Possible relationship of monocyte chemoattractant protein-1 with diabetic nephropathy. Kidney Int 58: 684-690, 2000.
39. Santos JC, de Brito CA, Futata EA, Azor MH, Orii NM, Maruta CW, Rivitti EA, Duarte AJS and Sato MN: Upregulation of chemokine $\mathrm{C}-\mathrm{C}$ ligand 2 (CCL2) and $\mathrm{C}-\mathrm{X}-\mathrm{C}$ chemokine 8 (CXCL8) expression by monocytes in chronic idiopathic urticaria. Clin Exp Immunol 167: 129-136, 2012.

40. Li W, Zhang Q, Wang M, Wu H, Mao F, Zhang B, Ji R, Gao S, Sun Z, Zhu W, et al: Macrophages are involved in the protective role of human umbilical cord-derived stromal cells in renal ischemia-reperfusion injury. Stem Cell Res (Amst) 10: 405-416, 2013.

41. Villanueva S, Ewertz E, Carrión F, Tapia A, Vergara C, Céspedes C, Sáez PJ, Luz P, Irarrázabal C, Carreño JE, et al: Mesenchymal stem cell injection ameliorates chronic renal failure in a rat model. Clin Sci (Lond) 121: 489-499, 2011.

42. Ezquer F, Giraud-Billoud M, Carpio D, Cabezas F, Conget P and Ezquer M: Proregenerative microenvironment triggered by donor mesenchymal stem cells preserves renal function and structure in mice with severe diabetes mellitus. Biomed Res Int 2015: 164703, 2015.

43. Bi B, Schmitt R, Israilova M, Nishio H and Cantley LG: Stromal cells protect against acute tubular injury via an endocrine effect. J Am Soc Nephrol 18: 2486-2496, 2007.

44. Zhang A, Wang Y, Ye Z, Xie H, Zhou L and Zheng S: Mechanism of TNF- $\alpha$-induced migration and hepatocyte growth factor production in human mesenchymal stem cells. J Cell Biochem 111: 469-475, 2010.

45. Kim YH, Ryu JM, Lee YJ and Han HJ: Fibronectin synthesis by high glucose level mediated proliferation of mouse embryonic stem cells: Involvement of ANG II and TGF- $\beta 1$. J Cell Physiol 223: 397-407, 2010.

46. Wise AF and Ricardo SD: Mesenchymal stem cells in kidney inflammation and repair. Nephrology (Carlton) 17: 1-10, 2012

47. Rasmusson I: Immune modulation by mesenchymal stem cells. Exp Cell Res 312: 2169-2179, 2006.

48. Meisel R, Zibert A, Laryea M, Göbel U, Däubener W and Dilloo D: Human bone marrow stromal cells inhibit allogeneic T-cell responses by indoleamine 2,3-dioxygenase-mediated tryptophan degradation. Blood 103: 4619-4621, 2004.

49. Tipnis S, Viswanathan C and Majumdar AS: Immunosuppressive properties of human umbilical cord-derived mesenchymal stem cells: Role of B7-H1 and IDO. Immunol Cell Biol 88: 795-806, 2010.

This work is licensed under a Creative Commons

Attribution-NonCommercial-NoDerivatives 4.0 International (CC BY-NC-ND 4.0) License. 